\title{
KOMMENTAR
}

\section{„Stuttgart 21“ - Krise oder Repolitisierung der repräsentativen Demokratie?}

\author{
Winfried Thaa
}

\begin{abstract}
"Stuttgart 21" - Crisis or Repoliticization of Representative Democracy?
Abstract: For many observers the fierce conflict concerning the infrastructure project "Stuttgart 21" indicates a crisis of representative democracy. Questioning common explanations of the conflict's escalation, the article develops the thesis that the present weakness in legitimating representative democracy should be explained as a result of severe deficits in its often overlooked configurative function. The latter consists in transforming differences and contradictions within society into political alternatives. More specifically the article states that the widespread discontent with representative democracy in Germany results from a contrary movement between the de-politicization of party competition on the one hand, and, stemming from civil society, a politicization of technical and infrastructural projects on the other.
\end{abstract}

Keywords: Democracy, Mass-Protests, Political Representation, Politicization

Schlagwörter: Demokratie, Massenproteste, Politische Repräsentation, Politisierung

\section{Krisendiagnosen: Schlechte Kommunikation? Mangelnde Führung? Zu wenig Responsivität?}

Die zugespitzten Auseinandersetzungen um den Abriss des alten und den Bau eines neuen, unter die Erde verlegten Bahnhofs in Stuttgart gelten als Symptom einer Krise der repräsentativen Demokratie. Dieser weitgehende Konsens unter den Kommentatoren findet allerdings schnell sein Ende, wenn es darum geht, genauer zu bestimmen, worin denn die Krise besteht und welche Lösungsmöglichkeiten sich anbieten. Auf den ersten Blick lassen sich mehrere, nicht ohne Weiteres miteinander zu vereinbarende Diagnosen unterscheiden: Der Konflikt gilt als Folge einer katastrophalen politischen Kommunikation der Repräsentanten und Projektträger mit den Bürgern (Brettschneider 2011a), als legitime Reaktion der Zivilgesellschaft auf die Verselbständigung der politischen Repräsentanten (Conradi 2010), aber auch umgekehrt, als Folge überzogener Erwartungen der Bürger (Münkler 2012) ${ }^{1}$ oder der Schwäche politischer Führung und ihrer zu großen

1 „Der, Wutbürger', von dem zuletzt häufiger die Rede war, ist das Produkt seiner eigenen überzogenen Erwartungen“ (Münkler 2012). 
Responsivität (Fliegauf u. Huhnholz 2011; allgemeiner Ritzi u. Schaal 2010). Noch grundsätzlicher, gewissermaßen aus einer evolutionären Perspektive, wird die viele Beobachter erstaunende Radikalität der Stuttgarter Bürger als Moment einer breiteren partizipatorischen Revolte gegen den Elitismus der repräsentativen Demokratie (Roth 2012) und für deren Ablösung durch Formen einer unmittelbaren Selbstbestimmung der Bürger (Ohme-Reinicke 2012) gedeutet.

Ich werde mich im Folgenden keiner dieser Interpretationen anschließen, sondern von einem normativen Verständnis repräsentativer Demokratie aus argumentieren, dass die genannten Diagnosen zu kurz greifen und, schlimmer noch, dazu beitragen können, die beklagte Krise zu verstärken. Den Grund hierfür sehe ich in einer unzulänglichen Konzeptualisierung politischer Repräsentation, die, von entgegengesetzten Seiten aus, die Gefahr birgt, deren konflikthaften, alternative Handlungsoptionen generierenden und sichtbar machenden Charakter zugunsten einer entpolitisierten Einheitsillusion zu schwächen. Die an den Auseinandersetzungen um Stuttgart 21 zu illustrierende These lautet, dass die derzeitige Krise politischer Repräsentation weder als ein Mangel an Responsivität noch als Führungsschwäche befriedigend erklärt werden kann. Sie folgt vielmehr aus Defiziten der in der Debatte meist übersehenen konfigurativen Funktion politischer Repräsentation, ihrer Aufgabe nämlich, gesellschaftliche Differenzen in politisch austragbare Konflikte zu transformieren. Spezifischer möchte ich behaupten, dass die derzeit weit über die Stuttgarter Auseinandersetzungen hinausreichende, an ähnlichen Großprojekten deutlich werdende Unzufriedenheit mit der repräsentativen Demokratie aus der gegenläufigen Bewegung zwischen einer während der letzten Jahrzehnte vonseiten der Parteieliten vorangetriebenen Entpolitisierung des Parteienwettbewerbs auf der einen sowie der zunehmenden Politisierung technischer und infrastruktureller Projekte auf der anderen Seite resultiert. Vor diesem Hintergrund werde ich aus den Ereignissen um „Stuttgart 21" vor allem zwei Schlüsse ziehen: Erstens, und das mag den Leser überraschen, ist das Bild, das die repräsentative Demokratie im Konfliktverlauf bietet, positiver als gemeinhin wahrgenommen. Den Gegnern des Projekts gelang es nicht nur, die von Landesregierung, Stadt und Bahn verfolgte Strategie der Alternativlosigkeit zu durchkreuzen. Die entstandene Konfliktkonstellation fand darüber hinaus, nicht zuletzt durch das Ergebnis der Landtagswahl 2011, auch Ausdruck auf der Ebene der politischen Repräsentation, was die derzeit populäre These ihres unaufhaltsamen Niedergangs zweifelhaft erscheinen lässt. Zweitens jedoch sprechen die auch im Stuttgarter Fall deutlich hervortretenden Schwierigkeiten, Konflikte um vermeintlich erforderliche Modernisierungsprojekte auf parlamentarischer Ebene adäquat zu repräsentieren, für die Einführung, bzw. den Ausbau institutionalisierter Möglichkeiten der Bürger, Beschlüsse der Parlamente aufzuschieben, öffentliche Auseinandersetzungen zu erzwingen und geplante Großprojekte damit auch gegen einen Elitenkonsens der politischen Meinungsbildung zu unterwerfen.

Obwohl es mir also weder um eine genaue chronologische Darstellung der Ereignisse, noch um eine eingehende sozialwissenschaftliche Analyse der hinter dem Projekt stehenden Interessen oder der dagegen protestierenden Gruppen geht, werde ich für eine Plausibilisierung meiner Überlegungen nicht umhin können, einige Grundzüge des Konflikts um „Stuttgart 21“ zu skizzieren. 


\section{Grundzüge des Konflikts um Stuttgart 21}

\subsection{Das repräsentative Verfahren: Erst entscheiden, dann ,kommunizieren}

In den Diskussionen um die politische Legitimation des Projekts Stuttgart 21 wird von seinen Befürwortern gern auf die demokratisch zustande gekommenen Beschlüsse der zuständigen Instanzen verwiesen. Tatsächlich wurde im November 1995 zwischen Bahn, Bund, Land, Region und Stadt eine Rahmenvereinbarung geschlossen, der der Stuttgarter Gemeinderat drei Wochen später zustimmte (Conradi 2010, S. 222). Wie eine Rekonstruktion der Genese des Konflikts in der Süddeutschen Zeitung beschreibt, ist das Projekt erstmalig im April 1994 auf einer Pressekonferenz von Oberbürgermeister Rommel, DB-Chef Dürr, Ministerpräsident Teufel und Bundesverkehrsminister Wissmann vorgestellt worden (Zielcke 2010a). Konkreter formuliert der Verfasser des Artikels, die Initiatoren hätten „unbemerkt von der Öffentlichkeit“ ihre „konzertierte Aktion seit längerem vorbereitet“ und eine „diebische Freude über ihren Überraschungscoup“ zu erkennen gegeben (Zielcke 2010a). Man darf hier wohl unterstellen, dass die Akteure davon ausgingen, ein für sich selbst sprechendes Vorhaben zu präsentieren und glaubten, für ihr effektives Zusammenwirken, mit dem sie das Projekt, wie es in solchen Fällen heißt, „auf den Weg brachten“, besonderes Lob zu verdienen. Ganz falsch lagen sie damit, zumindest hinsichtlich der Mehrheit der Parlamentarier in Stadtrat und Landtag, sicher nicht. Am 21. August 1996 kommentierte die Süddeutsche Zeitung: „Ohne eigenes Konzept, aber auch ohne denkbare Alternativen geprüft zu haben, hatten sich Stuttgarts Stadtväter vertraglich verpflichtet, das Projekt mitzutragen“ (zitiert nach Zielcke 2010a). Zwar gab es von Anfang an kritische Stellungnahmen ${ }^{2}$, und bereits 1995 wurde mit „Leben in Stuttgart Kein Stuttgart 21" die erste Bürgerinitiative gegen das Projekt gegründet, die breite Öffentlichkeit scheint das Vorhaben zunächst aber nicht empört zu haben (vgl. dazu Schlager 2010). Jedenfalls waren bereits Ende 1995 mit der Rahmenvereinbarung rechtlich die entscheidenden Würfel gefallen, ohne dass zuvor eine öffentliche Auseinandersetzung über die Wünschbarkeit des Projektes oder denkbare Alternativen stattgefunden hätte.

In diesem Zusammenhang wird zwar gern auf die bestehenden Beteiligungsmöglichkeiten der Bürger im Planfeststellungsverfahren verwiesen, in dem hier konkret mehr als 10.000 Eingaben und Einwände gemacht wurden (Zielcke 2010a). Allerdings kann dieses Verfahren ein Projekt lediglich in Einzelaspekten modifizieren, nicht aber insgesamt infrage stellen. Betrachtet man die Geschichte von „Stuttgart 21“, so erweist es sich geradezu als Idealtypus eines großen Infrastrukturprojektes, das unter den ,Machern' von Unternehmen und Exekutive konzipiert, ohne große Auseinandersetzungen durch die zuständigen Parlamente geschleust und dann einer breiteren Öffentlichkeit als alternativlos ,kommuniziert' wird.

2 Insbesondere ist hier das bereits 1995 veröffentlichte Buch von Winfried Wolf zu nennen. 


\subsection{Wirtschaftliche Notwendigkeiten und die Rhetorik der Alternativlosigkeit}

Die Befürworter des unterirdischen Neubaus führen vor allem zwei Hauptargumente ins Feld. Zum einen soll die Ersetzung des Kopfbahnhofs durch einen unterirdischen Durchgangsbahnhof eine infrastrukturelle Notwendigkeit darstellen, ohne die die verkehrsmäßig ungünstig liegende Wirtschaftsregion den Anschluss ans europäische Fernverkehrsnetz zu verlieren drohe. Bemerkenswert ist, dass die Befürworter des Vorhabens die bei Infrastrukturprojekten ohnehin übliche Rhetorik der Alternativlosigkeit in diesem Fall auf die Spitze trieben. Bahn, Stadt und Landesregierung ließen sich zu keinem Zeitpunkt auf eine Diskussion über Gegenvorschläge ein. Der bereits mehrfach zitierte Andreas Zielcke formuliert, dem Projekt sei vom ersten Tag an eine „absolutistische Ja/Nein Logik aufgebürdet“ gewesen. Entweder es komme zur geplanten Untertunnelung, oder alles bleibe beim Alten (Zielcke 2010a). Zum Zweiten, und hier liegt eine Besonderheit des Projektes, argumentieren seine Befürworter mit den einmaligen Chancen der Stadtgestaltung, die der Abbau mehrerer Hektar Gleisanlagen eröffnet. Erstaunlicherweise spielten die damit prinzipiell eröffneten Handlungs- und Gestaltungsmöglichkeiten in der öffentlichen Debatte jedoch kaum eine Rolle. Ein Hauptgrund hierfür ist darin zu sehen, dass die Stadt sich sehr früh auf Bebauungspläne festlegte, die der Bahn durch eine dichte Blockbebauung mit monofunktionalen Gebäuden für Büro- und Einkaufszentren einen hohen Grundstückserlös garantieren sollten (Schlager 2010, S. 117). Selbst die für Diskussionen über verschiedene Nutzungsoptionen eigentlich offene Seite des Projektes war damit ökonomischen Zwängen unterworfen und von Anfang an verbaut.

\subsection{Finanzielle Ungereimtheiten und "Maultaschen Connection“}

Obwohl enorme Kostensteigerungen und finanzielle Ungereimtheiten bei Großprojekten mittlerweile zum Alltag gehören, lässt sich über „Stuttgart 21 “ kaum schreiben, ohne dessen Verschwörungstheorien nahelegende Finanzierungsgeschichte zu erwähnen. Von besonderer Bedeutung aus Sicht der Gegner ist dabei der kurzfristige Planungsstopp des Projektes durch die Deutsche Bahn im Jahre 1999. Eine Prüfung durch das Unternehmen kam zum Ergebnis, das Projekt sei nicht komplett realisierbar. Darauf reagierte das Land mit einer Vorfinanzierung der ICE-Strecke zwischen Wendlingen und Ulm, und die Stadt Stuttgart kaufte der Bahn schon mal die Gleisanlagen ab, die durch den Bau des unterirdischen Bahnhofs frei werden sollten (Schlager 2010, S. 117). Die außerordentlich hohe Bereitschaft der politisch Verantwortlichen, enorme staatliche Mittel in die Vorfinanzierung des Projekts zu investieren, anhaltende Unsicherheiten in der Finanzierung hinzunehmen und immer wieder drastische Kostensteigerungen zu akzeptieren, lässt sich aus Sicht der Projektgegner nicht allein durch das öffentliche Interesse an einer (vermeintlich) besseren Verkehrslösung oder die sich durch das freiwerdende Areal bietenden Möglichkeiten der Stadtentwicklung erklären. Prominente Politiker und ihre Familienangehörigen sind als Berater von Firmen oder Mitglieder von Stiftungen an der geplanten Verwertung der frei werdenden Innenstadtflächen beteiligt (Schlager 2010, S. 133). Dieser hier nicht weiter auszuführende As- 
pekt legte es, insbesondere in einem seit vielen Jahrzehnten von derselben Partei regierten Bundesland, nahe, den Konflikt als Auseinandersetzung zwischen einer verfilzten, eigene Interessen verfolgenden Elite und der Mehrheit der normalen Bürger wahrzunehmen.

\subsection{Bürgerbegehren, Gerichtsurteile und Wahlen}

$\mathrm{Zu}$ einer ersten breiten Mobilisierung der Projektgegner kam es 2007 im Zuge eines von mehreren Organisationen und Bündnis 90/Die Grünen organisierten Bürgerbegehrens. Dabei gelang es innerhalb von sechs Wochen 67.000 Unterschriften zu sammeln. Der Stuttgarter Gemeinderat zeigte sich davon jedoch wenig beeindruckt und lehnte den beantragten Bürgerentscheid als unzulässig ab. Diese Entscheidung wurde später durch ein Urteil des Verwaltungsgerichtes bestätigt, mit der Begründung, ein Bürgerbegehren gegen einen bereits vollzogenen Gemeinderatsbeschluss sei unzulässig (Schlager 2010, S. 119). Vor dem Hintergrund dieser Auseinandersetzungen konnten Bündnis 90/Die Grünen als Gegner des Projektes bei den Stuttgarter Gemeinderatswahlen 2009 mit 25,9 Prozent der Stimmen zur stärksten Fraktion werden. Eine weitere, aus dem Protest gegen „Stuttgart 21“ entstandene Liste kam auf knapp fünf Prozent (Conradi 2010, S. 224).

\subsection{Wer demonstrierte?}

In Berichten und Diskussionsbeiträgen zu den Auseinandersetzungen um Stuttgart 21 wird immer wieder, teilweise erstaunt, teilweise aber auch empört, der bürgerliche Charakter der Demonstranten hervorgehoben. Dirk Kurbjuweit beschreibt den durch seinen Spiegel-Essay bekannt gewordenen „Wutbürger“ als „konservativ, wohlhabend und nicht mehr jung“ und macht unter den „Bürgerlichen, die da buhen, schreien und hassen “ sogar „CDU-Wähler und Rentner" aus (Kurbjuweit 2010, S. 26). Richtig ist daran, dass die soziale Zusammensetzung der Stuttgarter Demonstranten sich von früheren Protestbewegungen in der Bundesrepublik Deutschland stark unterschied. Zwei Studien, die eine durchgeführt vom WZB (Rucht et al. 2010), die andere vom „Göttinger Institut für Demokratieforschung “ (Göttinger Institut 2010), kommen bei leichten Abweichungen im Detail zum übereinstimmenden Ergebnis einer starken Überrepräsentation älterer, sehr gut ausgebildeter Demonstrationsteilnehmer. ${ }^{3}$ Nach der WZB-Studie waren über 60 Prozent der Demonstrierenden zwischen 35 und 64 Jahre alt, nur sieben Prozent jünger als 25 (Rucht et al. 2010, S. 3). Der Anteil der Befragten mit Hochschul- oder Fachhochschulabschluss lag mit knapp 50 Prozent mehr als doppelt so hoch wie im Bevölkerungsdurchschnitt (Rucht et al. 2010, S. 4). Herfried Münkler spricht von einer „demokratischen Oligarchie“, die sich auf die durch „Ausbildung und Beruf erworbenen Kompetenzen in Verbindung mit einem frei verfügbaren großen Zeitbudget“" stütze (Münkler 2010).

3 Ein zusammenfassender Vergleich der beiden Studien findet sich bei Kraushaar 2011. 
Das ganz normale Bürgertum war es aber nicht, das in Stuttgart Zeit und Kompetenzen einbrachte. Diejenigen, die noch bei der Landtagswahl 2006 CDU wählten, bildeten eine kleine Minderheit (weniger als 10 Prozent) unter den insgesamt überwiegend links und ökologisch orientierten Demonstrierenden (Göttinger Institut 2010, S. 16-17). Wolfgang Kraushaar erkennt aufgrund dieser Daten „in den Protestierenden vor allem die Repräsentanten der im Gefolge der 68er-Bewegung entstandenen links-alternativen Milieus“ und sieht sich an eine "Wanderdüne“ erinnert, die sich durch die Protestszenarien der vergangenen Jahrzehnte geschoben habe (Kraushaar 2011, S. 17).

\subsection{Massenmobilisierung, Polizeigewalt und Wir-Sie-Dichotomie}

In unserem Zusammenhang besonders interessant ist, dass auf die Frage nach dem Ereignis, das den Anlass bildete, sich gegen Stuttgart 21 zu engagieren, die weitaus größte Gruppe nicht den Beginn der Bauarbeiten 2010 nannte, sondern die Ablehnung des Bürgerentscheids 2007 (Rucht et al. 2010, S. 5). ${ }^{4}$ Demokratiedefizite bei der Planung des Projekts und im Umgang mit den Projektkritikern spielen auch bei den von den Befragten genannten Argumenten gegen das Projekt eine Hauptrolle (Rucht et al. 2010, S. 6). Die für den Widerstand gegen ein lokales Infrastrukturprojekt außergewöhnlich breite Mobilisierung mit den wöchentlichen Montagsdemonstrationen und zahlreichen Großdemonstrationen mit bis zu 80.000 Teilnehmern an Wochenenden lässt sich mit der unmittelbaren Betroffenheit durch die Baumaßnahmen und die verkehrspolitische Dimension des Projekts nicht erklären. Eher dürfte das Zusammentreffen einer hohen allgemeinen Unzufriedenheit mit dem Zustand der Demokratie in Deutschland (Göttinger Institut 2010, S. 13-14), der Empörung über das als undurchdringlicher Filz wahrgenommene Zusammenspiel von Politik, Wirtschaft und Justiz sowie einer als einseitig empfundenen Lokalpresse zu dieser weit über Baden-Württemberg hinaus Erstaunen auslösenden Mobilisierung geführt haben. Zorn über die Missachtung der Kritik eines großen Teils der Bürger mag auch erklären, warum es in den Auseinandersetzungen in Stuttgart zu einer ungewöhnlich starken Wir-Sie-Dichotomisierung kam. Jedenfalls lautete eine der am meisten gerufenen und auf Buttons angesteckten Parolen schlicht „Lügenpack“ - für eine überwiegend aus Freiberuflern, Lehrern und qualifizierten Angestellten bestehende Menge eine eher ungewöhnliche Äußerungsform. Die Politik der Landesregierung unter Ministerpräsident Mappus, der sich durch demonstrative Entschlossenheit und Stärke zu profilieren versuchte, begünstigte diese moralisierte Gegenidentität der Protestbewegung ebenso wie die Eskalation der Polizeigewalt im September 2010.

Im Mittelpunkt der Selbstbeschreibungen der aktiv an Protestmaßnahmen Beteiligten steht jedoch die Erfahrung eines in vielen Fällen als kreativ, einfallsreich

4 Insofern stimmt auch die wiederholt geäußerte Behauptung nicht, dass erst das Anrollen der Bagger den Protest ausgelöst und damit eine längst legitimierte Entscheidung viel zu spät in Frage gestellt worden wäre (etwa Vorländer 2011, S. 469). 
und befreiend empfundenen gemeinsamen Handelns. ${ }^{5}$ Allerdings gehört zum Stichwort Mobilisierung im Stuttgarter Fall auch eine im Verlauf des Konflikts zunehmende Polarisierung innerhalb der Bevölkerung, die ihren Ausdruck in Demonstrationen und Kundgebungen von Befürwortern des Projektes fand.

\subsection{Die Schlichtung - kompetenzlos aber folgenreich}

Über die Schlichtung Heiner Geißlers ließe sich ein extra Aufsatz schreiben. Andreas Zielcke nannte das Verfahren in der Süddeutschen Zeitung im doppelten Sinn postdemokratisch: zum einen, weil es der ursprünglich versäumten demokratischen Willensbildung nachgeschaltet war, zum anderen, weil die Gegner des Projekts den bereits über alle Rechtstitel zu seiner Durchsetzung verfügenden Betreibern gegenübersaßen, das Verfahren damit also eine Parodie auf einen herrschaftsfreien Diskurs darstellte (Zielcke 2010b). Als weiterer Kritikpunkt ist hinzuzufügen, dass das Verfahren und die Art seines Abschlusses nicht von den Beteiligten vorab festgelegt worden waren, sondern weitgehend Heiner Geißlers souveräner Entscheidung überlassen blieben (Rucht 2011). Dennoch leistete die Schlichtung etwas, das die vorausgegangene Willensbildung in den repräsentativen Organen von Stadt und Land sträflich vernachlässigt hatte. Sie inszenierte einen öffentlichen, von den Medien ausführlich wiedergegebenen und in der Bevölkerung breit rezipierten Streit insbesondere über die finanziellen und verkehrspolitischen Aspekte des Projekts. ${ }^{6}$ Ungeachtet der zugespitzten inhaltlichen Konflikte ging dabei offensichtlich vom Verfahren selbst eine stark befriedende Wirkung aus. Diese wusste Geißler mit seinem das Projekt unter Auflagen befürwortenden und damit als Kompromiss daherkommenden Schlichterspruch für einen Akzeptanzgewinn des Vorhabens zu nutzen. So verloren nach einer Untersuchung der Uni Hohenheim Bahn und Land während der Schlichtung mit ihren Positionen zur Verbesserung der Verkehrssituation, der Finanzierung und den Umweltfolgen in der befragten Öffentlichkeit zwar einige Prozent an Zustimmung. Das Verfahren insgesamt führte dennoch zu einem Meinungsumschwung zugunsten des Projekts (Brettschneider 2011b). ${ }^{7}$

\subsection{Regierungswechsel und Volksentscheid}

Die Auseinandersetzungen um „Stuttgart 21“ trugen dazu bei, durch die Landtagswahl im März 2011 die Dauerherrschaft der CDU in Baden-Württemberg zu beenden und die erste Landesregierung unter Führung der Grünen zu ermögli-

5 Annette Ohme-Reinicke versucht diesen Aspekt der Protestaktivitäten unter der in vielen Selbstbeschreibungen benutzten Metapher des „Aufwachens“ zu erfassen (Ohme-Reinicke 2012, S. 171-174).

$6 \mathrm{Zu}$ den Ergebnissen eines an der Universität Hohenheim durchgeführten Projekts zur Wahrnehmung und Bewertung der Schlichtung durch die Bevölkerung vgl. Brettscheider 2011b.

7 Die Hohenheimer Studie nennt als Hauptmotive hierfür die Milliardeninvestitionen, die durch das Projekt nach Baden-Württemberg fließen sollen, sowie die städtebaulichen Gestaltungsmöglichkeiten durch die frei werdenden Gleisflächen (Brettschneider 2011b, S. 213-214). Wie weit das von Geißler zur Begründung seines Schlichterspruchs hervorgehobene weit fortgeschrittene Stadium der Vorbereitungen für "Stuttgart 21“ und damit wiederum ein Sachzwang für diesen Meinungsumschwung den Ausschlag gab, sei dahingestellt. 
chen. Aufgrund einer Koalitionsvereinbarung zwischen Grünen und SPD fand dann im November 2011 in Baden-Württemberg die erste Volksabstimmung auf Landesebene statt, in der eine Mehrheit von 58,9 Prozent den Ausstieg des Landes aus dem Projekt ablehnte. Es liegt eine nicht geringe Ironie darin, dass die nachhaltigsten Effekte einer Bewegung, die unter ihren intellektuellen Interpreten vor allem wegen ihrer Spontaneität und Autonomie sowie ihrer antirepräsentativen Rhetorik geschätzt wurde ${ }^{8}$, in der Veränderung parlamentarischer Mehrheiten und der Durchsetzung einer Volksabstimmung bestanden.

\section{Lesarten des Konflikts}

\subsection{Zu wenig Führung, zu viel Responsivität?}

Eine der oben genannten Diagnosen zur Krise der repräsentativen Demokratie scheint bereits durch einen ersten Blick auf den Verlauf des Konflikts um „Stuttgart 21" widerlegt. Zu schwache politische Führung und eine zu hohe Responsivität der Repräsentanten (Stichwort „Stimmungsdemokratie“) lassen sich hier beim besten Willen nicht ausmachen. In Stuttgart erstaunt vielmehr die Beharrlichkeit, mit der die politischen Führungen von CDU und SPD gegen breiten Widerstand und nahezu ohne Rücksicht auf Stimmenverluste bei Gemeinderats- und Landtagswahlen am einmal gefassten Beschluss festhielten. Zudem scheiterte der wohl aus einer ähnlichen Krisendiagnose genährte Versuch des Ministerpräsidenten Mappus, sich als starke Führungspersönlichkeit zu profilieren, die das einmal als richtig Erkannte auch gegen Widerstände durchsetzt, geradezu kläglich.

Etwas mehr Plausibilität kann im Stuttgarter Fall ein formalistisches Repräsentationsverständnis, das auf die Autorisierung politischer Führung durch Wahlen abhebt, beanspruchen, wenn man aus ihm normative Erwartungen an das Verhalten der Bürger ableitet. Dann nämlich lässt sich mit dem Politikwissenschaftler Oscar Gabriel daran erinnern, dass „Partizipation ihren Platz in der repräsentativen Demokratie vornehmlich in Wahlen und im Vorfeld verbindlicher Entscheidungen hat" (Gabriel 2011). Dies ist im Kern die Position Joseph Schumpeters, der es für eine Erfolgsbedingung der Demokratie hält, dass die Wähler „einsehen, dass wenn sie einmal jemand gewählt haben, die politische Tätigkeit seine Sache ist und nicht die ihre" (Schumpeter 1942, S. 468). So gesehen erklärt sich die Eskalation des Konflikts aus dem Fehlverhalten der Bürger, ihrer Weigerung nämlich, die für eine repräsentative Demokratie konstitutive Arbeitsteilung zu akzeptieren. Die Forderung nach einer politischen Abstinenz der Bürger zwischen den Wahlen muss jedoch mindestens eine der folgenden Voraussetzungen machen: Entweder die Bürger haben gute Gründe, in den gewählten Politikern die Repräsentanten eines ihren Partikularinteressen übergeordneten Allgemeinwohls zu sehen oder sie akzeptieren deren Entscheidung als Folge eines Wahlergebnisses, das die Repräsentanten mit einem entsprechenden Mandat versah. Beides war in Stuttgart aber nun gerade nicht der Fall.

8 Beispielhaft dafür Ohme-Reinicke 2012. 
Dennoch lohnt es sich, das Anknüpfen an der elitistischen Demokratietheorie über den konkreten Fall hinaus etwas genauer zu betrachten. Eine ganze Reihe von Politikwissenschaftlern haben in jüngerer Zeit die Stärkung kompetenter Führung und die Personalisierung politischer Repräsentation als Ausweg aus der derzeitigen Krise der repräsentativen Demokratie empfohlen. ${ }^{9}$ Claudia Ritzi und Gary S. Schaal etwa argumentieren, die Stärkung politischer Führung könne

„dazu beitragen, notwendige[!] politische Entscheidungen zu implementieren und repräsentativ verfasste Demokratien aus der Handlungsunfähigkeit zu befreien, die aus Interessenkonflikten in pluralen Gesellschaften resultiert“ (Ritzi u. Schaal 2010, S. 9).

Das Zitat klingt zunächst einmal nach einer Neuauflage der bekannten konservativen Sorge um die Regierungsfähigkeit in pluralistischen Gesellschaften. Ritzi u. Schaal versuchen ihr jedoch eine neue Dringlichkeit zu verleihen, indem sie zum einen auf den Zerfall gesellschaftlicher Großmilieus und die daraus resultierende Fragmentierung politischer Präferenzen verweisen und zum anderen deren zunehmende Ökonomisierung behaupten. Im Ergebnis hätten wir es dann mit konsumorientierten Bürgern zu tun, die gegenüber der Politik eine Berücksichtigung ihrer im Kern unverhandelbaren Präferenzen einklagen. Dies führe dazu, „dass es für Parteien immer schwieriger wird, kohärente Programme mit ausgeprägtem inhaltlichen Profil für eine große Menge von Wählern anzubieten“ (Ritzi u. Schaal 2010, S. 12).

Was die genannten Funktionsdefizite der Parteien anbelangt, mag dies eine zutreffende Beschreibung darstellen. Mit ihrem Gewährsmann, dem ungarischen Politologen Körösényi, behaupten Ritzi und Schaal darüber hinaus jedoch eine Verschiebung des Ideals der Demokratie von der Nachfrage- zur Angebotsorientierung und „somit von einer Demokratie der Bürgerbeteiligung zu einer der Führungsorientierung“ (Ritzi u. Schaal 2010, S. 10). Ein „responsible government“, in der die „Regierung Verantwortung für Bürgerinnen und Bürger übernimmt, und zwar durch gute [!] Politik, die jedoch nicht an den Input des demokratischen Prozesses rückgekoppelt sein muss“ (Ritzi u. Schaal 2010, S. 11), soll die responsive Demokratie ersetzen.

Vielleicht wissen ja die Befürworter der in der deutschsprachigen Debatte verschämt „Leader Democracy“ genannten Führerdemokratie was „notwendige Entscheidungen“ sind und was eine „gute Politik“ ausmacht. Ihr Problem dürfte jedoch darin liegen, dass die Bürger, wie sich gerade in den Konflikten um Großprojekte zeigt, dazu unterschiedliche Meinungen vertreten. Deshalb kommt ihr Lösungsvorschlag nicht umhin, den Raum politischen Handelns von zwei Seiten aus zu schließen: Einerseits durch eine technokratische Output-Orientierung und andererseits durch die Reduktion des politischen Wettbewerbs auf massenmedial inszenierte Images des Führungspersonals. Zur Rechtfertigung dieser Beschränkung der politischen Partizipation der Bürger auf die Autorisierung ihrer Führer findet sich bei zeitgenössischen Autoren, im Gegensatz zu den klassischen Vertretern der Führerdemokratie in der ersten Hälfte des 20. Jahrhunderts, die

9 So etwa Körösényi 2005, Pakulski u. Higley 2008, Ritzi u. Schaal 2010. 
Behauptung, es gäbe im 21. Jahrhundert ohnehin nicht mehr viel politisch zu entscheiden. ${ }^{10}$

Man darf Zweifel haben, ob eine derart entpolitisierte Demokratie den politischen Repräsentanten die erforderliche Legitimation verschaffen kann. András Körösényi scheint dieses Problem bewusst zu sein. Er greift deshalb auf Carl Schmitts Begriff der „qualitativen Repräsentation“ zurück und grenzt diese, wie Schmitt selbst, gegenüber der profanen, quantitativen und mechanistischen Repräsentation des liberalen Parlamentarismus ab (Körösényi 2005, S. 375). ${ }^{11}$ Qualitative Repräsentation steht nach Schmitt nicht für ein Interesse oder eine Meinung, sondern ist nur als Verkörperung einer höheren Idee oder eines übergeordneten Werts durch eine Person möglich (Schmitt 1923, S. 36). Für Schmitt kann eine solche höhere Idee religiöser Natur sein, aber eben auch das durch Freund-Feind-Unterscheidung homogenisierte Volk. Damit sind wir schließlich bei der Einheitsverkörperung angelangt, dem logischen Fluchtpunkt eines auf die Autorisierung politischer Führung verkürzten Repräsentationsverständnisses. Während diese Einheit in der Variante der „sachkompetenten Führungspersönlichkeit" (Ritzi u. Schaal 2010, S. 9) in der Fiktion technischer und wirtschaftlicher Notwendigkeiten gründet, liegt sie im Fall einer Personalisierung übergeordneter Werte, wie sie Körösényi und Schmitt vorschwebt, im Charisma des Führers. In beiden Fällen bleibt von der Freiheit des Bürgers, politisch zu handeln, nur die Möglichkeit, zwischen den um Herrschaftspositionen konkurrierenden Führungsfiguren zu wählen.

\subsection{Zivilgesellschaft und responsive Demokratie}

Normativ unproblematischer und empirisch plausibler scheint der Versuch, die Stuttgarter Ereignisse durch die Brille deliberativer Demokratietheorien zu interpretieren und dann vor allem die mangelnde Responsivität der politischen Repräsentanten gegenüber der Zivilgesellschaft zu kritisieren. In diesem Sinn gibt Elisabeth Conradi in einem differenziert argumentierenden Aufsatz den von elitistischer Seite an die Protestierenden gerichteten Vorwurf einer Gefährdung der repräsentativen Demokratie an die politisch Verantwortlichen zurück (Conradi 2010). Zivilgesellschaft und Demokratie bedingten sich wechselseitig. Es sei die Aufgabe zivilgesellschaftlicher Assoziationen, gesellschaftliche Problemlagen „wie durch ein Megaphon“ öffentlich zu Gehör zu bringen (Conradi 2010, S. 227). Diesen Funktionen der Problemwahrnehmung, Artikulation und Lautverstärkung entspreche „auf der anderen Seite eine Regierung, die Anregungen aus der Zivilgesellschaft auch annimmt“ (Conradi 2010, S. 228). In diesem Demokratiemodell muss es der Zivilgesellschaft möglich sein, Probleme öffentlich so zu dramatisieren, „dass sie vom parlamentarischen Komplex übernommen und bearbeitet werden“" (Habermas 1992, S. 435).

10 Politische Führung besteht nach Ritzi und Schaal vor allem darin, „, to get the job done und bei den Bürgern Akzeptanz für die getane Arbeit zu generieren“ (Ritzi u. Schaal 2010, S. 12).

11 Vgl. dazu Carl Schmitt 1923. 
Gestützt auf das Schleusenmodell der deliberativen Demokratietheorie von Jürgen Habermas (1992, S. 429-467) konkretisiert Conradi drei Aufgaben, die im Verhältnis zwischen Zivilgesellschaft und repräsentativer Demokratie erfüllt werden sollten: Erstens gelte es, die Argumente der Bürger vor oder zu Beginn der parlamentarischen Willensbildung zu berücksichtigen, zweitens solle die Meinungs- und Willensbildung der Zivilgesellschaft den Prozess der parlamentarischen Entscheidungsfindung begleiten und drittens fungiere die Zivilgesellschaft auch als Korrektiv bereits getroffener Entscheidungen (Conradi 2010, S. 230). Vor dieser normativen Folie besteht das für die Eskalation des Stuttgarter Konflikts verantwortlich zu machende Fehlverhalten nicht in einer unzulässigen Überschreitung der Bürgerrolle durch die Protestierenden, sondern in den Responsivitätsdefiziten der politisch Verantwortlichen.

Der Interpretation Conradis liegt eine für deliberative Demokratietheorien konstitutive Verschiebung der Legitimitätsanforderungen an demokratische Politik zugrunde. Demokratische Legitimation wird nicht mehr allein, und vielleicht nicht einmal mehr in erster Linie, im Akt der Autorisierung der politischen Repräsentanten durch Wahlen gesehen, sondern in der Zugänglichkeit und Qualität öffentlicher Beratungsprozesse. Die repräsentative Demokratie ist demnach ein institutionelles Arrangement, das die Entscheidungsverfahren des politischen Systems in letzter Instanz an die kommunikative Meinungs- und Willensbildung der Öffentlichkeit zurückbinden soll. Als Legitimitätsbegründung ersetzt die Erwartung auf ein vernünftiges Ergebnis des Beratungsprozesses damit den durch Wahlen des Souveräns autorisierten Willen der Repräsentanten. ${ }^{12}$

$\mathrm{Zu}$ unterstreichen ist, dass Kommunikation bei Conradi und anderen deliberativen Theoretikern einen maßgeblich von unten nach oben, oder, in der Terminologie von Habermas, einen von der Peripherie ins Zentrum (Habermas 1992, S. 432) gerichteten Prozess meint und gerade nicht die seit einiger Zeit in Politik und Publizistik populäre, transitive Version, in der den Bürgern politische Entscheidungen ,kommuniziert werden'.

Dennoch kommt Conradi aufgrund ihres diskurstheoretischen Demokratiemodells zu einer positiven Einschätzung der Geißlerschen Schlichtung. Diese habe nicht nur zuvor unzugängliche Informationen veröffentlicht und eine komplexe Debatte zu den Vor- und Nachteilen des Projekts ermöglicht, sondern auch „das Publikum" im Sinne von Habermas ins Spiel gebracht, das die Chance bekam, „sich über all die vielen zur Diskussion stehenden Aspekte eine eigene Meinung bilden zu können“ (Conradi 2010, S. 231). In dieser positiven Bewertung der Stuttgarter Schlichtung, die eine Neuentscheidung ja gerade nicht ermöglichte, treten grundsätzliche Schwächen der deliberativen Perspektive besonders klar hervor. Ins-

12 Deliberative Theorien stimmen in der Ersetzung des Allgemeinwillens durch das Ergebnis einer möglichst inklusiven Deliberation überein, unterscheiden sich jedoch darin, ob bzw. wie weit sie den Deliberationsergebnissen Vernunftcharakter zuschreiben. Während Jürgen Habermas am kognitiven Charakter des Deliberationsprozesses und dem Vernunftanspruch demokratischer Entscheidungen festhält (etwa Habermas 1992, S. 613), verteidigt Bernard Manin die prinzipielle Kontingenz des Politischen und betont die Differenz zwischen logischen Beweisen und politischen Argumentationen. Letztere ließen dem Adressaten stets die Freiheit der Zustimmung oder Ablehnung (Manin 1987, S. 353). Vgl. dazu auch Thaa 2011, S. 100-127. 
besondere in ihrer diskurstheoretischen, auf die Legitimationskraft vernünftiger Ergebnisse setzenden Version von Habermas ignoriert die deliberative Demokratietheorie den agonalen Charakter politischer Auseinandersetzungen. Das Schleusenmodell der Demokratie von Jürgen Habermas unterstellt, Regierungsentscheidungen seien durch eine zivilgesellschaftlich generierte kommunikative Macht öffentlicher Vernunft zu unterwerfen. Politische Entscheidungen sollen durch die Meinungs- und Willensbildung „nicht nur nachträglich kontrolliert, sondern mehr oder weniger auch programmiert" werden (Habermas 1992, S. 364). Es gibt aber nicht die eine öffentliche Meinung, die von der Zivilgesellschaft ausgehend das staatliche Handeln lenken könnte. Das Stuttgarter Beispiel zeigt, dass auch eine sehr breite, publizistisch ungewöhnlich präsente Protestbewegung allenfalls eine Mehrheitsmeinung, nicht aber die öffentliche Meinung vertreten kann. Das deliberative Demokratiemodell neigt jedoch zur Einheitssuggestion auf der Seite von $\mathrm{Zi}$ vilgesellschaft und Öffentlichkeit. Sein Legitimitätsanspruch basiert auf der Unterstellung, die Kontingenz politischer Entscheidungen ließe sich durch den „Zwanglosen Zwang“ des besseren Arguments ersetzen. Da aber auch die bestinformierten und denkbar inklusivsten Beratungen nicht die eine richtige Lösung hervorbringen können, tendiert das deliberative Demokratiemodell dazu, das Herrschaftsmoment im Zustandekommen von Entscheidungen zu verschleiern. Gerade die befriedende Wirkung der Stuttgarter Schlichtung, in der es zwar nichts Grundsätzliches zu entscheiden gab, die dem Publikum aber effektiv den Eindruck vermittelte, dass beide Seiten ausgiebig zu Wort kommen konnten, belegt diese Gefahr. Sie verbarg strukturelle Ungleichheiten hinter dem kommunikativen Prozess des Austauschs von Argumenten und Gegenargumenten und ließ den willkürlichen Schiedsspruch Geißlers als letztlich zu akzeptierende Problemlösung erscheinen.

\subsection{Demokratie gegen Staat und Repräsentation?}

Gegen die letztgenannte Gefahr, eine Verschleierung von Herrschaftsverhältnissen, scheint die radikale Kritik repräsentativer Politik, die in der Stuttgarter Protestbewegung und ihren intellektuellen Interpreten auch vertreten war, gefeit zu sein. Aus dieser Sicht wenden sich die großen Protestbewegungen der letzten Jahre nicht nur gegen diesen oder jenen Missstand, sondern bilden zusammengenommen eine breite Revolte gegen das Repräsentationsprinzip als Kern der westlichliberalen Demokratien. ${ }^{13}$ Die Gegenüberstellung von repräsentativer und ,echter ${ }^{6}$ Demokratie trifft zweifellos ein wichtiges Moment der Selbstwahrnehmung der Teilnehmer an Protestbewegungen, die ihr selbstbestimmtes Handeln häufig der Tristesse im Normalbetrieb der repräsentativen Demokratie gegenüberstellen.

Annette Ohme-Reinicke hat dies in ihrem Buch zur Protestbewegung gegen Stuttgart 21 mit viel Emphase und wenig kritischer Distanz beschrieben, etwa wenn sie berichtet, „dass immer wieder Demonstranten ihre Freude darüber beschreiben, dass sich neue Freundschaften bilden, neue Netzwerke entstehen, dass die Menschen kommunikativer würden oder eben neuartige Beziehungen entstün-

13 So etwa Roth 2012 unter Berufung auf Hardt u. Negri 2011. 
den“ (Ohme-Reinicke 2012, S. 184). Derartige Erfahrungen ermöglichen ihr zufolge das „Erkennen der Illusion, das Subjekt könne sich politisch vertreten lassen“ (Ohme-Reinicke 2012, S. 172). Es gelte stattdessen, ein Verständnis von Bürgerschaft zu entwickeln, in dem „keine die Bürger stellvertretend repräsentierenden und entscheidenden politischen Organe, sondern die Bürger selbst es sind, die an öffentlichen Orten gemeinsam die Entscheidungen über die Gestaltung ihres Gemeinwesens treffen“ (Ohme-Reinicke 2012, S. 189).

Die im politischen Handeln gegen einen gemeinsamen Gegner erfahrene Freiheit zu beschreiben, ist eine Sache, eine andere jedoch, diese Erfahrung zur Grundlage eines neuen Demokratieverständnisses zu stilisieren. Letzteres erklärt nicht nur die performative Seite politischen Handelns, insbesondere die Erfahrungen der Selbstenthüllung, des Neuanfangs und der Gemeinsamkeit, zu seinem eigentlichen Sinn und muss dann gegebenenfalls in Kauf nehmen, dass die Protestaktionen zu einer vorrangig selbstbezüglichen, nur noch um ihrer selbst willen betriebenen Aktivität werden. Darüber hinaus tendieren die Verklärungen von Protestbewegungen zur Grundlage einer neuen, unmittelbare Selbstbestimmung ermöglichenden Demokratie aber auch dazu, sich selbst als das Ganze zu setzen. So argumentiert OhmeReinicke, dass eine wahrhaft republikanische Bürgerlichkeit ohne alle Repräsentationsorgane „kommunitär, als wirkliche Bewegung agierend, umgesetzt werden kann“ (Ohme-Reinicke, S. 189). Ähnlich erhoffen sich Michael Hardt und Antonio Negri in den bewusst auf Repräsentation verzichtenden, horizontal partizipatorischen Strukturen der Occupy-Bewegung das Modell einer „wirklichen Demokratie“ (Hardt u. Negri 2011). Eine solche Hypostasierung der gegen einzelne Missstände kämpfenden Bewegungen zum eigentlichen Ort der Demokratie untergräbt jedoch die Pluralität des politischen Raumes und endet als bloße Kehrseite der zu Recht kritisierten technokratischen Entpolitisierung.

Auf theoretisch anspruchsvollerem Niveau finden derartige Verklärungen von Protestbewegungen eine Entsprechung in poststrukturalistisch geprägten Theorien einer „radikalen Demokratie“ (Laclau u. Mouffe 1985), einer „rebellierenden“ (Abensour 2012) oder, wie es Rancière formuliert, einer Demokratie der „Opposition zweier Logiken" (Rancière 2002, S. 110). Gemeinsam ist diesen Theorien, dass sie das Politische und in der Folge die Demokratie gleichsetzen mit dem kollektiven Widerstand gegen einen hegemonialen Diskurs oder die Ordnung der Herrschaft. Demokratische Politik besteht dann in einem Akt des Bruchs mit der gesellschaftlichen Ordnung der Ungleichheit (Rancière 2002), oder sie besteht im Aufrechterhalten einer Zäsur gegenüber den institutionalisierten Formen der politischen Konkurrenz im demokratischen Staat (Abensour 2012).

Was an dieser Sichtweise befremdet, ist nicht die Betonung der konstitutiven Rolle des Konflikts für demokratische Politik. Politisches Handeln, das heißt Handeln in einem durch Kontingenz bestimmten Raum, ist tendenziell immer auch ein Handeln gegen Andere. Befremdlich ist auch nicht der dem politischen Handeln von diesen Autoren zugeschriebene transzendierende Charakter. Mit Hannah Arendt ließe sich argumentieren, dass jedes politische Handeln einen Neuanfang setzt und damit Gegebenes überschreitet (Arendt 1981, S. 164-243). Befremden muss vielmehr, dass diese Theorien, geprägt von ihren poststrukturalistischen Grundlagen, die Optionalität politischen Handelns zu einer dichotomi- 
schen Logik verengen und auf einen totalisierenden Gestus der Negation festlegen. Sie konfrontieren Hegemonie und Gegenhegemonie (Laclau u. Mouffe 1985), Herrschaft und Volk (Rancière 2002, S. 24) oder auch gleich ganz konkret Reiche und Arme (Rancière 2002, 24). Damit jedoch bleibt das Bekenntnis dieser Theorien zu Nicht-Identität und Vielfalt vordergründig. Es erschöpft sich im Insistieren auf einem unaufhebbaren Gegensatz und in der Parteinahme für den Protest, die Negation, den Bruch. Weit davon entfernt, damit einen gemeinsamen politischen Raum aufzuspannen, in dem verschiedene Handlungsalternativen repräsentiert werden und auf vielfältige Weise um Unterstützung werben können, impliziert allein schon die Rede von dem Volk und der Herrschaft die Rekonstruktion essentialistischer Kollektivsubjekte auf den beiden entgegengesetzten Polen der Dichotomie. ${ }^{14}$

\subsection{Differenzrepräsentation und plurales politisches Handeln}

Vor dem Hintergrund der kritisierten wichtigsten Lesarten des Konflikts lassen sich nun die eingangs formulierten Thesen ausführlicher begründen. So gegensätzlich die verschiedenen Interpretationen in ihren politischen Schlussfolgerungen auch sein mögen, gemeinsam ist ihnen eine unangemessene Konzeptualisierung des Konflikts in der Beziehung zwischen Bürgern und politischen Repräsentanten. Die elitistische Demokratietheorie versucht Protestbewegungen, zumindest nach gefallenen Entscheidungen, unter Hinweis auf den Autorisierungsakt demokratischer Wahlen weitgehend zu delegitimieren. Deliberative Demokratietheorien dagegen rationalisieren Konflikte zu einem Moment der öffentlichen Meinungs- und Willensbildung, die von responsiven politischen Repräsentanten aufgegriffen und in eine vernünftige Ergebnisse sichernde Programmierung des politischen Systems umgesetzt werden soll. Die Interpretationen schließlich, die sich von Protestbewegungen eine neue, direkte Form der Demokratie erwarten, stilisieren sie zum Bruch mit der bestehenden Ordnung, sie lokalisieren sie also außerhalb der Repräsentationsbeziehung. Eine Alternative zur Delegitimierung, Rationalisierung und Externalisierung des Konflikts lässt sich jedoch gewinnen, wenn wir politische Repräsentation als Differenzrepräsentation verstehen.

Dass Repräsentation Differenz impliziert und das Ideal einer größtmöglichen Identität zwischen Repräsentanten und Repräsentierten deshalb verfehlt ist, wusste bereits Hanna Pitkin in ihrem klassischen Werk zum Begriff der Repräsentation. ${ }^{15}$ Im deutschsprachigen Raum benutzte Ernst Vollrath den Begriff der Differenzrepräsentation im Anschluss an Claude Leforts Kritik totalitärer Identitätsrepräsentation und betont mit ihm die für Verfassungsdemokratien konstitutive Unterscheidung zwischen Regierung und Regierten (Vollrath 1992, S. 76). Neuere Repräsentationstheorien nehmen dieses antitotalitäre Moment auf, indem sie Re-

14 Ausführlich zu den Schwächen der poststrukturalistisch geprägten radikalen Demokratietheorien, die rechtlichen und institutionellen Bedingungen politischer Freiheit zu denken vgl. Volk 2013 (in diesem Heft).

$15, \ldots[. .$.$] representation seems to require a certain distance or difference as well as resemblance or$ correspondence" (Pitkin 1967, S. 68). 
präsentation dezidiert gegen eine Logik der Identität abgrenzen, unterstreichen dabei jedoch stärker als etwa Vollrath die partizipatorischen Potenziale der durch politische Repräsentation mehrfach implizierten Differenzen. ${ }^{16}$ Denkt man Repräsentation als Differenzbeziehung, so gilt dies in mindestens dreifacher Hinsicht: für die Beziehung zwischen den Abgeordneten und ihren Wählern, für die zwischen den Bürgern sowie schließlich für diejenige zwischen den Repräsentanten. Für alle drei Beziehungen sei Differenz konstitutiv und unaufhebbar. In unserem Zusammenhang entscheidend ist nun, dass durch diese Verabschiedung des Ideals der möglichst exakten Repräsentation einer gegebenen Einheit oder eines vorab gebildeten Willens, Agonalität und Partizipation (theoretisch) in die Repräsentationsbeziehung hineingeholt werden. Denn aus der Anerkennung einer unaufhebbaren vertikalen Differenz zwischen den Repräsentanten und den Repräsentierten folgt logischerweise eine für alle Akteure geltende prinzipielle Offenheit der Frage, was und wie repräsentiert werden sollte. Wenn es kein gesichertes Wissen über das zu Repräsentierende geben kann, dann rücken Meinungsverschiedenheiten und Konflikte ins Zentrum aller drei genannten Beziehungsdimensionen.

Ob man diese Politisierung der Repräsentationsbeziehungen durch Anerkennung einer unaufhebbaren Differenz mit Derridas Poststrukturalismus (Young 1997), mit einer postmodernen Ästhetik (Ankersmit 1996), durch eine ideengeschichtliche Genealogie politischer Repräsentation (Urbinati 2006a) oder ganz ohne metatheoretische Vergewisserung aus einer Analyse zeitgenössischer Demokratien (Plotke 1997) begründet, macht im Ergebnis wenig Unterschied: Die Nichtidentität in der Repräsentationsbeziehung wird zur Voraussetzung für die Pluralität und Optionalität des Politischen. Daraus folgt dann aber auch, öffentliche Kontroversen, Protestaktionen und in letzter Instanz auch Formen des zivilen Ungehorsams nicht als Gefährdung der repräsentativen Demokratie zu verstehen, sondern als Momente eines verschiedene Partizipationsformen ermöglichenden, konflikthaften politischen Prozesses, der erst durch demokratische Repräsentation ermöglicht wird.

Ein Missverständnis wäre es allerdings, den politischen Repräsentanten dabei lediglich die Aufgabe eines responsiven Aufgreifens der zivilgesellschaftlichen Meinungs- und Willensbildung zuzuschreiben. Politische Repräsentation erschöpft sich auch nicht in der Widerspiegelung gegensätzlicher gesellschaftlicher Meinungen und Interessen. Politische Repräsentation hat vielmehr eine konstitutive Funktion für die von ihr Repräsentierten, und erst Repräsentation ermöglicht die kollektive Einwirkung der Bürger auf sich selbst. Dazu etwas ausführlicher Iris Marion Young:

\footnotetext{
"There is no constituency prior to the process of representation, no people who form an original unity they then delegate onto the derivative representative. Without the motive of a political decision deferred onto another who is accountable to them through public procedures, 'the people' might not go looking for each other in order to form a base of public opinion and account" (Young 1997, S. 359).
}

Spezifischer besteht die konfigurative Funktion politischer Repräsentation darin, die Vielfalt gesellschaftlicher Interessen und Meinungen an konkurrierende Inter-

16 Zu einem Überblick zu dieser Debatte vgl. Thaa 2008. 
pretationen von politischen Prinzipien (wie Freiheit, Gleichheit oder Gerechtigkeit) und gesellschaftlichen Werten (wie Wirtschaftswachstum, Sicherheit, Partizipation) anzubinden. Erst dadurch können aus der Vielfalt gesellschaftlicher Interessen und Meinungen politisch entscheidbare Alternativen werden. Und erst durch die dauerhafte Präsenz der konfligierenden Prinzipien und Werte in der politischen Arena bilden sich zeitlich stabile Referenzpunkte, die in umgekehrter Richtung auf die gesellschaftliche Meinungsbildung und die Formierung von Gruppen zurückwirken. ${ }^{17}$ In diesem Sinn sieht Nadia Urbinati eine der Hauptaufgaben repräsentativer Politik darin, der Gesellschaft eine komplexe Struktur „of meanings and interpretations of citizens ${ }^{6}$ beliefs and opinions about what their interests are" zu geben (Urbinati 2006b, S. 28).

In der jüngeren europäischen Geschichte war es vor allem der Konflikt zwischen Arbeit und Kapital, der durch die Bildung entsprechender Parteien eine dauerhafte Repräsentation innerhalb des politischen Systems fand. Marcel Gauchet bezeichnet diesen „Einzug des gesellschaftlichen Konflikts ins Innere des politischen Systems“ als „demokratisches Wunder“ (Gauchet 1990, S. 199). Die herausragende Leistung der modernen Demokratie besteht demnach in der Repräsentation des gesellschaftlichen Konflikts innerhalb des politischen Systems. Diese Leistung ist nicht als ein einmaliger Vorgang - etwa dem Aufstieg der Arbeiterparteien - zu sehen, sondern als ein andauernder Prozess, in dem Modifikationen der alten, aber auch neue Konflikte ihre politischen Repräsentationen finden.

\section{4. „Stuttgart 21“ und die Möglichkeiten zur Repolitisierung politischer Repräsentation}

Aufgrund eines solchen Repräsentationsverständnisses lässt sich die zunehmende Politikverdrossenheit der letzten Jahrzehnte zumindest teilweise als Reaktion auf die Unfähigkeit oder auch den fehlenden Willen der großen Volksparteien erklären, den weiter bestehenden gesellschaftlichen Widerspruch zwischen Kapital und Arbeit politisch zu repräsentieren. Statt diesen Widerspruch auf widerstreitende Interpretationen von Prinzipien und Werten zu beziehen und daraus Handlungsalternativen $\mathrm{zu}$ formulieren, die den Bürgern Diskussion, Urteilsbildung und Entscheidungen ermöglichen, konzentriert sich die Konkurrenz zwischen den Parteien auf medienvermittelte Images und Kompetenzzuschreibungen ihres Spitzenpersonals.

In dieser ohnehin stark entpolitisierten Situation zeichnet sich die Konstellation zu Beginn der Auseinandersetzungen in Stuttgart durch die Weigerung beider großer Parteien aus, das Projekt des Bahnhofneubaus überhaupt als eine politische, also im Konflikt widerstreitender Prinzipien und Werte zu entscheidende Angelegenheit zu betrachten. Die große Mehrheit der Mandatsträger in Stadtrat und Landtag versteht ihr Mandat als Autorisierung zum technokratischen ,Durchregieren'. Sieht man von den oben erwähnten Partikularinteressen der Entscheidungsträger ab, so findet die Entpolitisierung im Fall von „Stuttgart 21“, wie bei anderen Großprojekten auch, ihre stärkste Rechtfertigung in der (Selbst-)Ver-

17 Vgl. dazu auch in einer Interpretation der Schriften Claude Leforts: Weymans 2006, S. 190. 
pflichtung der Politik auf einen wirtschaftsfreundlichen Wachstumskurs. Stichworte wie „Sicherung des Wirtschaftsstandorts“, „Stärkung der regionalen Wirtschaftskraft“, „Modernisierung der Infrastruktur“ „Schaffen von Arbeitsplätzen“ u. ä. m., beschwören nicht so sehr eine politische Gemeinsamkeit (die ja auch erst einmal gefunden werden müsste), sondern verleihen den jeweiligen Projekten vielmehr eine höhere, der Politik übergeordnete Legitimität. Ob Flughafenerweiterung oder Bahnhofsneubau - was für das zukünftige Wohlergehen der Wirtschaft notwendig erscheint, wird in der Regel dem politischen Konflikt vorausgesetzt.

Demgegenüber führt der Verlauf des Konflikts in Stuttgart vor Augen, dass ein erheblicher Teil der Bevölkerung - und zwar nicht zufällig die besser gebildeten und wohlhabenderen Bürger - nicht mehr bereit ist, die bei Infrastrukturprojekten üblichen Strategien der Entpolitisierung zu akzeptieren. Der als „Wutbürger“ geschmähte Zeitgenosse zeichnet sich nicht, wie Herfried Münkler unterstellt (Münkler 2012), durch eine quasi kindliche Anspruchshaltung gegenüber der Politik aus. Vielmehr klagt er die Politisierung von Entscheidungen ein und beurteilt ihre Folgen nach seinen Vorstellungen einer lebenswerten Stadt oder Region. Republikanisch gesprochen geht es um das „gute Leben“, das im Gegensatz zur vermeintlich alternativlosen ökonomischen Notwendigkeit immer verschieden interpretiert werden kann. Konkret scheiterte die Entpolitisierungsstrategie von Politik und Bahn im Falle von „Stuttgart 21“ an einfachen und deshalb auch von manchem intellektuellen Beobachter belächelten Fragen: Rechtfertigen wenige Minuten Zeitersparnis auf der Strecke Stuttgart-Ulm die Zerstörung eines einmaligen, in der Innenstadt gelegenen Parks? Ist die höhere Nahverkehrskapazität des alten Bahnhofs nicht wichtiger als die Beschleunigung auf den Fernstrecken? Braucht Stuttgart ein neues Büro- und Bankenviertel?

Zur Zuspitzung in den Stuttgarter Auseinandersetzungen kam es, weil die Parteien nicht in der Lage waren, die hinter derartigen Fragen stehende Konfliktlinie angemessen zu repräsentieren. Gegen diese These ließe sich auf die Wahlerfolge der Grünen verweisen und einwenden, dass diese aufgrund der Unterstützung der Protestbewegung bereits 2009 zur stärksten Fraktion des Stuttgarter Gemeinderats wurden. In der Tat erwies sich die parteipolitische Repräsentation, besonders wenn man die Ablösung der CDU als Dauerregierungspartei in Baden-Württemberg berücksichtigt, durchaus als reaktionsfähig. Allerdings zog sich dieser Vorgang über Jahre hin, und trotz der spektakulären Stimmengewinne der Grünen war das einmal beschlossene Vorhaben nicht mehr zu verhindern. In der Regel, und das gilt trotz des günstigen Umfelds selbst für den Stuttgarter Fall, können Protestbewegungen gegen einzelne Großprojekte kaum hoffen, die Mehrheitsverhältnisse auf parlamentarischer Ebene kurzfristig zu verändern. Dies macht es erforderlich, nach Möglichkeiten zu suchen, wie die Bürger einen bestehenden Konsens zwischen wirtschaftlichen und politischen Eliten durchbrechen und gegen deren Strategien der Entpolitisierung bereits in der Planungsphase von Projekten eine öffentliche Auseinandersetzung erzwingen können.

Da die Parteien aufgrund sozio-kultureller Veränderungen immer weniger in der Lage zu sein scheinen, ihre konfigurative Funktion wahrzunehmen und gesellschaftliche Konflikte auf der repräsentativen Ebene darzustellen, scheint es sinnvoll, die „negative Macht der Bürger“ (Urbinati 2006a, S. 217) zu stärken, indem 
man Vetomöglichkeiten einführt, die es erlauben, politische Entscheidungen der Repräsentanten zu verzögern, mit Alternativen zu konfrontieren, einer öffentlichen Debatte zu unterwerfen und gegebenenfalls auch durch Volksabstimmungen zu entscheiden. Das Ziel ist dabei nicht, die Fiktion eines unmittelbaren Willens des Volkes an die Stelle der Repräsentanten zu setzen, sondern einen Weg zu eröffnen, gesellschaftlich umstrittene, in den bestehenden Machtkonstellationen aber nur unzureichend repräsentierte Fragen zu politisieren. Neben den bereits existierenden juristischen Einspruchsmöglichkeiten unmittelbar Betroffener sollten deshalb zu frühen Zeitpunkten des Planungs- und Entscheidungsprozesses auch politische Einspruchsmöglichkeiten der Bürger geschaffen werden. ${ }^{18}$

Die Zukunft der Demokratie hängt davon ab, ob es gelingen kann, den aus Sicht der Bürger wichtigsten Fragen und Konflikten der gesellschaftlichen Entwicklung wieder die Gestalt demokratisch entscheidbarer Alternativen zu geben. Ohne eine Wiederbelebung politischer Repräsentation wird das nicht zu leisten sein.

\section{Literatur}

Abensour, Miguel. 2012. Die rebellierende Demokratie. Blätter für deutsche und internationale Politik 5:90-98

Ankersmit, Frank R. 1996. Aesthetic Politics. Political Philosophy Beyond Fact and Value. Stanford: Stanford University Press.

Arendt, Hannah. 1981. Vita Activa. München: Piper.

Brettschneider, Frank. 2011a. Kommunikation und Meinungsbildung bei Großprojekten. Aus Politik und Zeitgeschichte 44-45/2011:40-47.

Brettschneider, Frank. 2011b. Die Schlichtung zu „Stuttgart 21“: Ein Prototyp für die Bürgerbeteiligung bei Großprojekten? Forum Stadt. Vierteljahreszeitschrift für Stadtgeschichte, Stadtsoziologie, Denkmalpflege und Stadtentwicklung 38:203-218.

Conradi, Elisabeth. 2010. Zivilgesellschaft und Demokratie. Die Bedeutung der Öffentlichkeit im Konflikt um das Infrastrukturprojekt ,Stuttgart 21'. Zeitschrift für politische Theorie 1:221-232.

Fliegauf, Marc T., und Sebastian Huhnholz. 2011. Parlamentarische Führung. Aus Politik und Zeitgeschichte 4/2011: 21-26.

Gabriel, Oscar. 2011. Die SPD fährt einen verhängnisvollen Schlingerkurs. Interview mit Bettina Wieselmann, http://www.tagblatt.de/Home/nachrichten/ueberregional/alleszum-bahnprojekt-stuttgart-21_artikel,-Stuttgart-21-Die-SPD-faehrt-einen-verhaengnisvollen-Schlingerkurs-_arid,114688.html. Zugegriffen: 30.07.2012.

Gauchet, Marcel. 1990. Tocqueville, Amerika und Wir. Über die Entstehung der demokratischen Gesellschaften. In Autonome Gesellschaft und libertäre Demokratie, Hrsg. Ulrich Rödel, 123-206. Frankfurt a. M.: Suhrkamp.

$18 \mathrm{Ob}$ auch kooperative und konsultative Beteiligungsformen, wie Bürgerhaushalte, Planungszellen u. ä. m., in denen die Bürger über keine Entscheidungskompetenzen verfügen, zu einer Politisierung im obigen Sinn beitragen können, oder aber die elitären und expertokratischen Züge der Politik weiter verstärken, hängt von ihrer konkreten Ausgestaltung ab. Zur Diskussion dieser Formen im einzelnen vgl. etwa Urbinati u. Warren 2008; Geißel 2011; Linden u. Thaa 2011. 
Geißel, Brigitte. 2011. Partizipative Innovationen auf lokaler Ebene - Chance oder Gefahr für die politische Repräsentation von Armen und Fremden? In Krise und Reform politischer Repräsentation, Hrsg. Markus Linden und Winfried Thaa, 195-216. Baden-Baden: Nomos.

Göttinger Institut für Demokratieforschung. 2010. Neue Dimensionen des Protests? Ergebnisse einer explorativen Studie zu den Protesten gegen Stuttgart 21. http://www. demokratie-goettingen.de/content/uploads/2010/11/Neue-Dimensionen-des-Protests. pdf. Zugegriffen: 25.05.2012.

Habermas, Jürgen. 1992. Faktizität und Geltung. Beiträge zur Diskurstheorie des Rechts und des demokratischen Rechtsstaates. Frankfurt a. M.: Suhrkamp.

Hardt, Michael, und Antonio Negri. 2011. The Fight for Real Democracy at the Heart of Occupy Wall Street. http://www.foreignaffairs.com/articles/136399/michael-hardt-andantonio-negri/the-fight-for-real-democracy-at-the-heart-of-occupy-wall-street. Zugegriffen: 06.08.2012.

Körösényi, András. 2005: Political Representation in Leader Democracy. Government and Opposition 40:358-378.

Kraushaar, Wolfgang. 2011. Protest der Privilegierten? Oder was ist wirklich neu an den Demonstrationen gegen Stuttgart 21? Mittelweg 36:5-22.

Kurbjuweit, Dirk. 2010. Der Wutbürger - Stuttgart 21 und die Sarrazin-Debatte: Warum die Deutschen so viel protestieren. DER SPIEGEL 41/2010:26-27.

Laclau, Ernesto, und Chantal Mouffe. 1985. Hegemony and Socialist Strategy. Towards a Radical Democracy, London: Verso.

Linden, Markus, und Winfried Thaa. 2011. Die Krise der Repräsentation - gibt es Auswege? In dies.: Krise und Reform politischer Repräsentation, 305-324. Baden-Baden: Nomos.

Manin, Bernard. 1987. On Legitimacy and Political Deliberation. Political Theory 15:338-368.

Münkler, Herfried. 2010. Der gereifte Demonstrant - Regieren und Partizipieren in einer alternden Gesellschaft. http://www.dradio.de/dlf/sendungen/hintergrundpolitik/ 1349007/. Zugegriffen: 24.7.2012.

Münkler, Herfried. 2012. Die Verdrossenen und die Empörten. Steht die Demokratie am Scheideweg. http://www.nzz.ch/aktuell/feuilleton/uebersicht/die-verdrossenen-und-dieempoerten-1.16611315. Zugegriffen: 30.07.2012.

Ohme-Reinicke, 2012. Das große Unbehagen. Die Protestbewegung gegen "Stuttgart 21": Aufbruch zu neuem bürgerlichen Selbstbewusstsein? Stuttgart: Schmetterling Verlag.

Pakulski, Jan, und John Higley. 2008. Toward Leader Democracy? In Public Leadership. Perspectives and Practices, Hrsg. Paul 't Hart und John Uhr. 43-53. Canberra: Australian National University Press.

Pitkin, Hanna Fenichel. 1967. The Concept of Representation. Berkeley/Los Angeles: University of California Press.

Plotke, David. 1997. Representation is Democracy. Constellations 4:19-34.

Rancière, Jacques. 2002. Das Unvernehmen. Politik und Philosophie. Frankfurt a. M.: Suhrkamp.

Ritzi, Claudia, und Gary S. Schaal. 2010. Politische Führung in der Postdemokratie. Aus Politik und Zeitgeschichte 2-3/2010:9-15.

Roth, Roland. 2012. Vom Gelingen und Scheitern sozialer Bewegungen. Forschungsjournal Neue soziale Bewegungen 25:21-31. 
Rucht, Dieter. 2011. Der Geißlersche Schlichtungsprozess: Legitimation durch Verfahren oder Neutralisierung von Kritik? http://www.kopfbahnhof-21.de/index.php?id=691. Zugegriffen: 21.01.2011.

Rucht, Dieter, Britta Baumgarten, Simon Teune und Wolfgang Stuppert. 2010. Befragung von Demonstranten gegen Stuttgart 21 am 18.10.2010. http:/www.wzb.eu/sites/default/files/projekte/stgt_21_kurzbericht_2010.pdf. Zugegriffen: 30.06.2012.

Schlager, Alexander. 2010. Die Proteste gegen "Stuttgart 21". Sozial. Geschichte Online 4: 112-137. http://duepublico.uni-duisburg-essen.de/servlets/DerivateServlet/Derivate25690/08_Schlager\%20Stuttgart.pdf. Zugegriffen: 25.07.2012.

Schmitt, Carl. 1923. Römischer Katholizismus und politische Form. Stuttgart: Klett-Cotta.

Schumpeter, Joseph A. 1942. Kapitalismus, Sozialismus und Demokratie. München: Franke 1980.

Thaa, Winfried. 2008. Kritik und Neubewertung politischer Repräsentation. Politische Vierteljahresschrift 49:618-640.

Thaa, Winfried. 2011. Politisches Handeln. Baden-Baden: Nomos.

Urbinati, Nadia. 2006a. Representative Democracy. Principles and Genealogy. Chicago: University of Chicago Press.

Urbinati, Nadia. 2006b. Political Representation as a Democratic Process. In Redescriptions 10:18-40.

Urbinati, Nadia, und Marc E. Warren. 2008. The Concept of Representation in Contemporary Political Theory. Annual Review of Political Science 11:387-412.

Volk, Christian. 2013. Zwischen Entpolitisierung und Radikalisierung. Zur Theorie von Demokratie und Politik in Zeiten des Widerstands. Politische Vierteljahresschrift 54:75-110.

Vollrath, Ernst. 1992. Identitätsrepräsentation und Differenzrepräsentation. Rechtsphilosophische Hefte 1:65-78.

Vorländer, Hans. 2011. Der Wutbürger. In Ideenpolitik. Geschichtliche Konstellationen und gegenwärtige Konflikte, Hrsg. Harald Bluhm, Karsten Fischer und Marcus Llanque, 467-478. Berlin: Akademie-Verlag.

Weymans, Wim. 2006. Freiheit durch politische Repräsentation - Lefort, Gauchet und Rosanvallon über die Beziehung zwischen Staat und Gesellschaft. In Die unendliche Aufgabe, Hrsg. Reinhard Heil und Andreas Hetzel, 185-207. Bielefeld: transcript.

Wolf, Winfried. 1995. „Stuttgart 21 “. Hauptbahnhof im Untergrund? Köln: Neuer ISP Verlag. Young, Iris Marion. 1997. Deferring Group Representation. In Ethnicity and Group Right, Hrsg. Ian Shapiro und Will Kymlicka, 349-376. New York: New York University Press.

Zielcke, Andreas. 2010a. Geistige Kessellage. „Der große Wurf“ und das kleine Zeitfenster: Warum Stuttgart 21 an einem unheilbaren Mangel leidet. Ein überfälliger Rückblick. Süddeutsche Zeitung 19.10.2010: 13.

Zielcke, Andreas. 2010b. Schlichtung und Wahrheit, Süddeutsche Zeitung 03.12.2010: 15.

\section{Autorenangaben}

Prof. Dr. Winfried Thaa,

Universität Trier, Fachbereich III - Politikwissenschaft, Universitätsring 15, 54286 Trier, thaa@uni-trier.de 